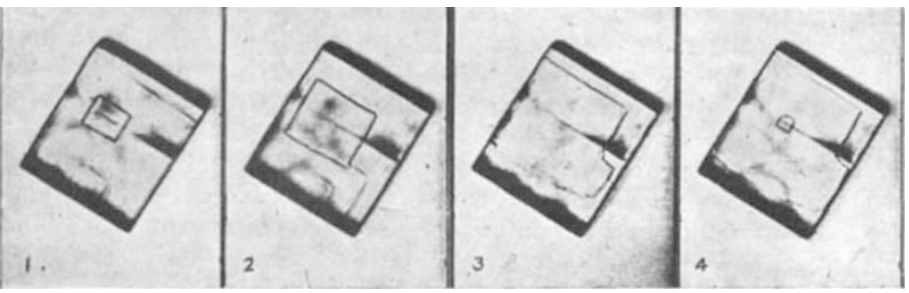

Spread of one crystallization layer (frames 1 to 3) and the start of the next (frame 4) on a sodium chloride crystal growing in the presence of cadmium ions. Frames were cut from cinematograph fllm at 2-sec. intervals. Original magniflcation $\times 50$, enlarged $\times 3$ photographically. Transmitted light

where the magnetostriction $\lambda=2 \times 10^{-5}$, and the saturation intensity $J_{s}=1,100$ c.G.s. units. The stresses $\sigma$ due to magnetostriction should not exceed $\lambda E$, and Young's modulus $E=2 \times 10^{12}$ dynes cm. ${ }^{-2}$. This gives $\sigma=4 \times 10^{7}$ dynes $\mathrm{cm} .^{-2}$, and an increase of coercive force of less than 1 oersted.

A reduction of 825 gauss in the remanence of a bar of 'Alcomax II' hardened without a field has been produced by a longitudinal pressure of $2.2 \times 10^{\circ}$ dynes $\mathrm{cm} .^{-2}$, suggesting that the increase in remanence of, say, 2,500 gauss produced by magnetic hardening would require

that most dyes which modify the habit of a crystal are not adsorbed when present in the solution in concentrations below a minimum value. What this value is for the inorganic ions in question cannot easily be determined by the radioactive tracer technique, which reveals only the fractional amount adsorbed.

That cadmium and lead are more strongly adsorbed on sodium chloride than the other ions need not indicate a fundamental difference in the adsorption mechanism. Royer ${ }^{4}$, discussing habit modification, suggested an explanation for the assumed adsorption of cadmium, zinc and manganese chlorides. These three salts form rhombohedral crystals which consist of alternate layers of anions and cations in planes parallel to the (111)-face. On this face the lattice mesh is a rhombohedron the sides of which are 3.85 A. for cadmium chloride, 3.77 A. for zinc chlor ide and $3 \cdot 70 \mathrm{~A}$. for manganese chloride. Sodium chloride also consists of alternate layers of anions and cations parallel to the (111)-face, and on this face the lattice mesh is a rhombohedron the sides of which are $3.97 \mathrm{~A}$. Royer attributed the adsorption to the good fit of these planes. That cadmium chloride has the best fit is compatible with the evidence pointing to its stronger adsorption.

A detailed study of the incorporation of radioactive tracer lead and cadmium ions into sodium chloride crystals has been made and will be published else. where.

Chemistry Branch,

А. Н. Воотн

Atomic Energy Project,

National Research Council,

Chalk River, Ontario. Jan. 30.

${ }^{1}$ Bunn, C. W., and Emmett, H., Discuss. Faraday Soc., No. 5, 119 (1949).

${ }^{2}$ Kading, H., Z. phys. Chem., A, 162, 174 (1932).

srance, W. G., in "Colloid Chemistry", 5, 443-456 (ed. J. Alexander,

- Royer, L., C.R. Acad. Sci., Paris, 198, 585 (1934).

\section{Preferred Domain Orientation in Permanent Magnet Alloys}

THE properties of 'Alcomax II' cooled with and without a magnetic field have been compared by Hoselitz and McCaig ${ }^{1}$. The improvement in useful properties, which is due to a preferred domain orientation, is explained according to Bozorth ${ }^{2}$ by the 'freezing in' of magnetostrictive stresses. However, magnetic hardening may increase the coercive force by up to 100 oersteds, while the increase due to magnetostrictive stresses should be of the order $\lambda \sigma / J_{s}$, a stress of $6 \times 10^{9}$ dynes $\mathrm{cm}^{-2}$, that is, 150 times that expected from Bozorth's mechanism.

Stoner and Wohlfarth' have worked out in detail the magnetic properties of an agglomeration of singledomain ellipsoidal particles. This theory could account for the properties of 'Alcomax', and is important in connexion with certain powder magnets but there is as yet no direct evidence for such particles in cast magnets, and no mechanism has been suggested by which a magnetic field could so control the growth of precipitates of a second phase as to produce oriented particles. (Note added during revision: Kittel, Nesbitt and Shockley ${ }^{4}$ have now suggested a possible mechanism, comments on which will appear elsewhere.) In any such mechanism the maximum energy available is $J_{s} H$, which, taking $H=1,000$, and $J_{s}=400$ at the high temperatures involved, cannot exceed $4 \times 10^{5}$ ergs $\mathrm{cm}^{-2}$, a figure which seems small compared with the energies usually involved in phase changes in metals.

An incipient phase change might produce large stresses, random in direction and limited by the possibility of plastic flow. With positive magnetostriction, if the direction of magnetization is parallel to a compressive stress or perpendicular to a tensile stress, an energy term $\lambda \sigma$ due to the interaction of the stress and magnetization will arise, provided the field is sufficient to align the direction of magnetization against the action of the stress. This energy of interaction might have a trigger-like action promoting the relief along certain preferred slip planes of much larger stresses produced during precipitation. Those stresses, tension parallel and compressions perpendicular to the field, which would produce the actual domain orientation, would tend to be retained while others would be relieved. Mechanical equilibrium could be maintained by other stresses in a second phase with a smaller intensity or magnetostriction. Alternatively, the production of slip planes with a preferred orientation might influence the further course of precipitation so as to produce the effect of oriented particles.

I thank Dr. K. Hoselitz and Mr. F. R. N. Nabarro for valuable suggestions and criticisms which $I$ have endeavoured to meet so far as possible.

Permanent Magnet Association,

M. McCaIG

Central Research Laboratory, Brown Street,

Sheffield 1.

Feb. 6.

1 Hoselitz, K., and McCaig, M., Nature, 164, 581 (1949).

Bozorth, R. M. and Dillinger, J. F., Physics, 6, 285 (1935)

s Stoner, E. C., and Wohlfarth, E. P., Phil. Trans. Roy. Soc., 240, 599 (1948)

4 Kittel, Nesbitt and Shockley, Phys. Rev., 77, 839 (1950). 